\title{
REPRESENTATION OF A GROUP AS A TRANSITIVE PERMUTATION GROUP
}

BY G. A. MILLER

Let $G$ be any group of finite order $g$ and let $H$ be any subgroup of order $h$ contained in $G$. If the operators of $G$ are separated into right or into left augmented co-sets with respect to $H$ and if these $g / h=n$ co-sets are then multiplied successively on the right or on the left respectively by the various operators of $G$, they will be permuted as units according to a transitive permutation group $T$ which is simply isomorphic with the quotient group of $G$ with respect to the largest invariant subgroup of $G$ which appears in $H$, if $H$ is not itself invariant under $G$. If $H$ is invariant under $G$, then $T$ will be a regular group which is simply isomorphic with $G / H$. The case when $H$ is non-invariant under $G$ and does not involve any invariant subgroup of $G$ besides the identity is especially important since $T$ is then simply isomorphic with $G$, as was pointed out for right co-sets by W. Dyck in 1883.

If $K$ is any subgroup of $G$ which has operators in each of the co-sets of $G$ with respect to $H$ and if $K_{0}$ is the cross-cut of $H$ and $K$, then $K_{0}$ may be invariant under $K$ or it may involve an invariant subgroup under $K$. If one of these conditions is satisfied, $H$ must involve a subgroup which is invariant under $G$ and includes this invariant subgroup under $K$. This follows directly from the facts that this invariant subgroup is transformed into all of its conjugates under $G$ by operators of $H$ and that a complete set of conjugate subgroups always generates an invariant subgroup if it does not generate the entire group. We have then the following result.

THEOREM 1. If a group $G$ is separated into co-sets with respect to a subgroup $H$ and if another subgroup $K$ has operators in each of these co-sets, then the largest invariant subgroup under $K$ which appears in the cross-cut of $H$ and $K$ is contained in an invariant subgroup of $G$ which is found in $H$.

In particular, when $T$ is simply isomorphic with $G$, then the largest invariant subgroup under $K$ which appears in the crosscut of $H$ and $K$ is the identity. 
A necessary and sufficient condition that a subgroup of $G$ corresponds to a transitive subgroup of degree $n$ in $T$ is that it has operators in each of the co-sets of $G$ with respect to $H$. In the case when $T$ is simply isomorphic with $G$ this transitive subgroup of degree $n$ must also be simply isomorphic with the corresponding subgroup of $G$. We have then the following result.

THEOREM 2. When $G$ is represented as a simply isomorphic transitive permutation group of degree $n$ with respect to a subgroup $H$, then a necessary and sufficient condition that a given subgroup of $G$ corresponds to a simply isomorphic transitive subgroup of degree $n$ is that operators of this subgroup of $G$ appear in each of the co-sets of $G$ with respect to $H$.

When $T$ is not simply isomorphic with $G$, a subgroup of $G$ is not necessarily simply isomorphic with the corresponding subgroup of $T$. A necessary and sufficient condition that such a simple isomorphism exists is that this subgroup of $G$ has only the identity in common with the largest invariant subgroup of $G$ which is found in $H$. When $H$ is invariant under $G$, then the regular group $T$ will correspond to every subgroup of $G$ whose operators are distributed among all of the co-sets of $G$ with respect to $H$. Subgroups of $G$ whose operators are not thus distributed will correspond to regular constituents of the subgroups of $T$. A necessary and sufficient condition that a subgroup of $G$ corresponds either to a regular subgroup of $T$ or to a regular constituent of a subgroup of $T$ is that its cross-cut with $H$ is invariant under this subgroup.

Suppose that $G$ involves a subgroup $H$ whose operators are distributed among all except one of the co-sets of $G$ with respect to $H$ and that $T$ is simply isomorphic with $G$. The subgroup $H$ must correspond to a simply isomorphic subgroup of $T$ which is of degree $n-1$ and transitive on these $n-1$ letters. This subgroup must therefore appear in a conjugate of the subgroup of $T$ which corresponds to $H$. It therefore results that $T$ is multiply transitive. Moreover, when $T$ is multiply transitive, $G$ must contain such a subgroup.

THEOREM 3. A necessary and sufficient condition that a group $G$ appears as a multiply transitive group when it is represented as a transitive permutation group with respect to a subgroup $H$ is that at least one subgroup of $G$ has its operators distributed among all except one of the co-sets of $G$ with respect to $H$. 
From this theorem it results directly that a necessary and sufficient condition that a group is $r$-fold transitive when it is represented as a simply isomorphic transitive group with respect to a subgroup $H$ is that one can find $r-1$ successive subgroups $H_{1}, H_{2}, \cdots, H_{r-1}$ each of which after the first is contained in the preceding and has its operators distributed among all except one of the co-sets of $G$ with respect to $H$ in which the operators of the preceding subgroup are found, the operators of $H_{1}$ appearing in all these co-sets except one. It may be noted that the operators of all of these $H$ 's have the property that their products into any co-set which contains no operator of the corresponding $H$ appear in this co-set.

Suppose that all the operators of $G$ are distributed successively with respect to a subgroup $H$ of $G$ both into right and also into left co-sets. It is easy to verify that every such right co-set is identical with some left co-set of $G$ with respect to a conjugate of $H$ and that the totality of the right co-sets of $H$ includes left co-sets of $G$ with respect to all the conjugates of $H$ under $G$. Similarly, the totality of the left co-sets with respect to $H$ includes right co-sets with respect to all the conjugates of $H$ under $G$. This results directly from the theorem that the multiplying operators of co-sets can always be so chosen that the totality of the right multipliers is identical with the totality of left multipliers. This proves the following theorem.

THEOREM 4. The right co-sets of any group $G$ with respect to a given subgroup $H$ are composed of left co-sets of $G$ with respect to all the conjugates of $H$ under $G$, and vice versa.

If more than one right co-set of $G$ with respect to $H$ is equal to a left co-set with respect to the same conjugate of $H$, the number of such right co-sets is equal to the index of $H$ under the largest subgroup of $G$ in which $H$ is invariant. In particular, this number is an invariant of $G$. A necessary and sufficient condition that only one right co-set of $G$ with respect to $H$ is equal to a left co-set with respect to a given conjugate of $H$ is that $H$ is transformed into itself only by its own operators under $G$. This is also a necessary and sufficient condition that only one left co-set of $G$ with respect to $H$ is equal to a right co-set with respect to a given conjugate of $H$. The operators of $G$ which when multiplied on the right into a given right co-set of $G$ with respect to $H$ have all their products in this co-set constitute the 
conjugate of $H$ in the equivalent left co-set, and vice versa.

A necessary and sufficient condition that $T$ is simply transitive is that at least one conjugate of $H$ under $G$ has its operators distributed among less than $n-1$ co-sets of $G$ with respect to $H$. In particular, when these operators are distributed among $n-2$ such co-sets $n$ must be even and $T$ must involve a system of imprimitivity composed of $n / 2$ sets of letters. This is also a necessary and sufficient condition that $H$ is invariant under a subgroup of $G$ whose order is exactly $2 h$. If $G$ is simply isomorphic with $T$ and a subgroup of $G$ has its operators distributed among $n-2$ of the co-sets of $G$ with respect to $H$, its order cannot exceed $2 h$, and when it has this order it must involve a subgroup of index 2 which is conjugate with $H$ under $G$. Moreover, $H$ corresponds to a transitive subgroup of degree $n-2$ in $T$.

The University of Illinois

\section{A NOTE ON TRANSFINITE ORDINALS}

\section{BY BEN DUSHNIK}

In a supplementary note to an article of theirs, ${ }^{*}$ Alexandroff and Urysohn demonstrated the following theorem.

If to every ordinal $\alpha$ of the second class there corresponds an ordinal $\mu(\alpha)$ such that $\mu(\alpha)<\alpha$, then there exists a non-denumerable set of ordinals of the second class

$$
\alpha_{1}, \alpha_{2}, \cdots, \alpha_{n}, \cdots, \alpha_{\omega}, \cdots, \alpha_{\lambda}, \cdots
$$

such that

$$
\mu\left(\alpha_{1}\right)=\mu\left(\alpha_{2}\right)=\cdots=\mu\left(\alpha_{\lambda}\right) \cdots .
$$

The present note applies a different method to prove the following more general result.

Theorem. Let $\Omega_{\delta}$ be the smallest ordinal whose power is $\boldsymbol{N}_{\delta}$, where $\delta>0$ is a non-limiting ordinal. If to every transfinite ordinal $\alpha<\Omega_{\delta}$ there corresponds an ordinal $\mu(\alpha)$ such that $\mu(\alpha)<\alpha$,

* Mémoire sur les espaces topologiques compacts, Verhandelingen of the Amsterdam Academy, (1), vol. 45, No. 1. 